\title{
Web-scale Multimedia Search for Internet Video Content
}

\author{
Lu Jiang \\ School of Computer Science \\ Carnegie Mellon University \\ Pittsburgh, PA 15213 \\ lujiang@cs.cmu.edu
}

\begin{abstract}
The Internet has been witnessing an explosion of video content. According to a Cisco study, video content is estimated to account for $80 \%$ of all the entire world's internet traffic by 2019 . Video data are becoming one of the most valuable sources to assess information and knowledge. However, existing video search solutions are still based on text matching (text-to-text search), and could fail for the huge volumes of videos that have little relevant metadata or no metadata at all. The need for large-scale and intelligent video search, which bridges the gap between the user's information need and the video content, seems to be urgent.

In this thesis, we propose an accurate, efficient and scalable search method for video content. As opposed to text matching, the proposed method relies on automatic video content understanding, and allows for intelligent and flexible search paradigms over the video content, including textto-video and text\&video-to-video search. Fig. 1 illustrates an example. Suppose our goal is to search the videos about birthday party. In traditional text-to-text queries, we have to search the keywords in the user-generated metadata (titles or descriptions). In a text-to-video query, however, we might look for visual clues in the video content such as "cake", "gift" and "kids", audio clues like "birthday song" and "cheering sound", or visible text like "happy birthday". See Fig. 1(a) Text-to-video queries are flexible and can be further refined by Boolean and temporal operators. After watching the retrieved videos, the user may select a few interesting videos to find more relevant videos like these. This can be achieved by issuing a text\&video-to-video query which adds the selected video examples to the query. See Fig. 1(b)

The proposed method provides a new dimension of looking at content-based video search, from finding a simple concept like "puppy" to searching a complex incident like "a scene in urban area where people running away after an explo-
\end{abstract}

\footnotetext{
* This work was partially supported by the Intelligence Advanced Research Projects Activity (IARPA) via Department of Interior National Business Center contract number D11PC20068.
}

Permission to make digital or hard copies of part or all of this work for personal or classroom use is granted without fee provided that copies are not made or distributed for profit or commercial advantage and that copies bear this notice and the full citation on the first page. Copyrights for third-party components of this work must be honored. For all other uses, contact the owner/author(s)

WSDM 2016 February 22-25, 2016, San Francisco, CA, USA

(C) 2016 Copyright held by the owner/author(s).

ACM ISBN 978-1-4503-3716-8/16/02

DOI: http://dx.doi.org/10.1145/2835776.2855081

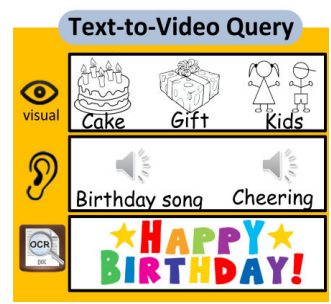

(a) text-to-video

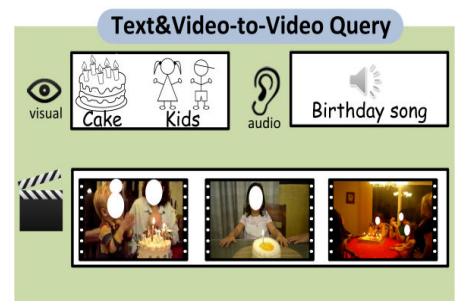

(b) text\&video-to-video
Figure 1: Proposed queries on "birthday party".

sion". To achieve this ambitious goal, we propose several novel methods focusing on accuracy, efficiency and scalability in the novel search paradigm. First, we introduce a novel self-paced curriculum learning theory that allows for training more accurate semantic concepts. Second, we propose a novel and scalable approach to index semantic concepts that can significantly improve the search efficiency with minimum accuracy loss. Third, we design a novel video reranking algorithm that can boost accuracy for video retrieval.

The extensive experiments demonstrate that the proposed methods are able to surpass state-of-the-art accuracy on multiple datasets. In addition, our method can efficiently scale up the search to hundreds of millions videos, and only takes about 0.2 second to search a semantic query on a collection of 100 million videos, 1 second to process a hybrid query over 1 million videos. Based on the proposed methods, we implement E-Lamp Lite, the first of its kind large-scale semantic search engine for Internet videos. According to National Institute of Standards and Technology (NIST), it achieved the best accuracy in the TRECVID Multimedia Event Detection (MED) 2013, 2014 and 2015, the most representative task for content-based video search. To the best of our knowledge, E-Lamp Lite is the first contentbased semantic search engine that is capable of indexing and searching a collection of 100 million videos.

\section{Categories and Subject Descriptors}

H.3.1 [Information Storage and Retrieval]: Content Analysis and Indexing; H.3.3 [Information Search and Retrieval]: Search process; I.2.10 [Vision and Scene Understanding]: Video analysis

\section{Keywords}

Big Data; Web Search; Video Content Search; Contentbased Retrieval; Multimedia Event Detection 